BULLETIN Bulletin hispanique

HispaniquE Université Michel de Montaigne Bordeaux

112-1| 2010

Actes du Colloque « langue, littérature, littéralité »

\title{
Autour du discours polémique en Nouvelle Espagne
}

Yves Aguila

\section{(2) OpenEdition}

Journals

Édition électronique

URL : http://journals.openedition.org/bulletinhispanique/1145

DOI : 10.4000/bulletinhispanique. 1145

ISSN : $1775-3821$

Éditeur

Presses universitaires de Bordeaux

Édition imprimée

Date de publication : 1 juin 2010

Pagination : 287-308

ISBN : 978-2-86781-692-5

ISSN : 0007-4640

Référence électronique

Yves Aguila, "Autour du discours polémique en Nouvelle Espagne », Bulletin hispanique [En ligne], 112-1 | 2010, mis en ligne le 01 juin 2013, consulté le 26 avril 2019. URL : http://

journals.openedition.org/bulletinhispanique/1145; DOI : 10.4000/bulletinhispanique.1145

Tous droits réservés 


\title{
Autour du discours polémique en Nouvelle Espagne
}

\author{
Yves Aguila \\ Université Michel de Montaigne Bordeaux
}

À la fin de la période coloniale, au Mexique, les vieux antagonismes entre créoles et Espagnols péninsulaires śexacerbent en même temps que naissent de nouvelles polémiques, autour de la prononciation, par exemple, ou suscitées par certains "proyectos", ces avatars au XVIII de l'arbitrisme des siècles antérieurs. De propos délibéré, on n'en a retenu ici que les expressions les plus pittoresques et les formes les plus originales : "décimas" mises au service de linjure, parodie des langages populaires, tant urbains que ruraux ou indiens, critique par l'absurde des mécanismes de pensée des "proyectistas".

A fines del periodo colonial, los antiguos antagonismos entre criollos y españoles peninsulares se exacerban al tiempo que nacen nuevas polémicas, en torno a la pronunciación, por ejemplo, o provocadas por ciertos "proyectos», esos avatares en el siglo XVIII del arbitrismo de los siglos anteriores. Deliberadamente, sólo se han conservado aqui sus expresiones más pintorescas y sus formas más originales: décimas puestas al servicio de la injuria, parodia de los lenguajes populares, tanto urbanos como rurales, reducción al absurdo de los mecanismos del razonamiento proyectista.

At the end of the Colonial era in Mexico, old antagonisms between peninsular Spanish and Creoles are aggravated, as new polemics arise - on pronunciation for instance or provoked by some 'proyectos', those eighteenth century avatars of the arbitrism from earlier centuries. Deliberately, we only retained here the most picturesque expressions and the most original forms : "décimas» in the service of abuse, parody of popular language - urban as well as rural or Indian -, criticism of the mechanisms of thought of the 'proyectistas' by reducing them to the absurd.

Mots-clés: Créoles - Gachupines - Polémique - Prononciation-Proyectismo.

$B H i$, Tome 112, no 1 - juin 2010 - p. 287 à 308. 


\begin{abstract}
$\Lambda$ la fin de la période coloniale, au Mexique, le social et le politique fournirent des sujets de controverse qui, le plus souvent, bénéficièrent du canal que proposait la naissance, en 1805, d'un quotidien dont les rédacteurs furent souvent à l'origine de polémiques qui présentaient l'avantage intéressant de leur fournir de la copie. Ce que je propose ici, c'est de voir, à travers quelques exemples, les ressources utilisées pour l'expression des opinions critiques et antagoniques à l'époque, quelques unes des formes empruntées par le discours polémique. En d'autres termes, d'aller faire un petit tour au magasin de l'histoire pittoresque.

Les discussions qui eurent lieu reflètent souvent, même quand le sujet paraît anodin et superficiel, des antagonismes profonds, entre créoles et gachupines, bien sûr, mais aussi entre conservateurs - aussi bien créoles que péninsulaires - et adeptes d'une modernisation souvent liée à une aspiration patriotique ou nationaliste.
\end{abstract}

\title{
GaChupines ET CRÉOLES
}

C'est le plus ancien des antagonismes puisque sa naissance doit coïncider avec celle du premier créole. Loin de s'atténuer avec le temps, plus la colonie approche de sa fin et plus il s'exacerbe, malgré les efforts du pouvoir espagnol pour purger le vocabulaire de ces deux termes et les remplacer par ceux d' "Espagnols américains » et "Espagnols européens ». La volonté manifeste de réduire la différence d'origine géographique à un simple accident par l'adjectif pour souligner la communauté substantive d'appartenance à une même entité politique ne rencontra aucun écho.

$\mathrm{Au}$ premier rang des innombrables motifs de vindicte des créoles, trônait le mépris ostensible qu'affichaient les péninsulaires à leur sujet, un comportement attesté par les voyageurs étrangers qui remarquèrent à l'unisson que le plus miteux des commis espagnols se croyait issu de la cuisse d'Hernán Cortés. Voilà pourquoi, sous les espèces de la fable, se défoula poétiquement l'humiliation éprouvée devant des attitudes aussi injustes que blessantes. Un émule local des Samaniego et autres Iriarte opposa donc dans une longue fable intitulée El coyote y la raposa la généreuse hospitalité créole au dédain insupportable de leurs hôtes. La fable raconte l'histoire d'un coyote créole qui accueille une renarde venue d'Espagne pour devenir sa femme. On y décrit tout ce que le gentil coyote met en œuvre pour satisfaire sa promise, depuis les moyens de transport rustiques qu'il met à sa disposition jusqu'à la fête qu'il donne en son honneur. Ainsi, on précise que 


\title{
AUTOUR DU DISCOURS POLÉMIQUE EN NOUVELLE ESPAGNE
}

\author{
Eran los instrumentos \\ una cierta figura de atabales \\ compuestos de canillas de animales: \\ zapatearon el tango, \\ la bamba y el fandango, \\ sin faltar el jarave \\ ni todo cuanto cabe \\ en un festín alegre y razonado
}

Beaucoup d'entrain et de couleur locale pour rien, puisque, nous dit le fabuliste :

$$
\begin{aligned}
& \text {... la novia a todo muestra enfado, } \\
& \text { afeando el sarao y concurrencia. }
\end{aligned}
$$

Là, ç'en est trop et le coyote hors de lui, après lui avoir fait remarquer que

$$
\begin{aligned}
& \text { el obsequio de aquesta compañia, } \\
& \text { que usted tanto critica y escarnece, } \\
& \text { es un efecto de cortesanía, } \\
& \text { y por fin es lo que el país ofrece }
\end{aligned}
$$

lui annonce qu'il annule le mariage et après un discours vengeur la renvoie dans ses foyers, seule, détestée de tous, rabo entre piernas et criblée d'adjectifs rien moins que flatteurs et bons à prendre pour tous les gachupines: ridiculo, odioso, necio, altiva, vana, melindrosa. La fameuse "visée de disqualification ", caractéristique reconnue du discours polémique par les spécialistes en la matière, est on ne peut plus manifeste. Ce qui l'est moins, c'est une motivation cachée, car la fable, en fait, était revancharde à un autre niveau. Dans la réalité, en effet, les mariages intergroupes se faisaient surtout dans l'autre sens et une grande spécialité gachupina était le proverbial et imagé braguetazo, c'est-à-dire les épousailles d'un fringant péninsulaire avec une riche héritière créole, sous le nez et au grand dépit des prétendants autochtones.

Comme il se doit en un temps où elle était omniprésente, la sphère religieuse ne fut pas épargnée. Déjà la religion à la mexicaine avait fait l'objet de remarques acerbes, dans les années 1785, de la part d'un Espagnol établi au Mexique du nom d'Hipólito Villaroel. Les processions, en particulier, avaient excité sa verve tant elles étaient loin de se dérouler avec tout le recueillement qu'on était en droit d'en attendre. Même pour des jours aussi solennels que le Vendredi Saint, elles retentissaient des cris des vendeurs 
de fruits, de boissons, de crécelles, etc ${ }^{1}$. En matière d'ambiance, la palme semblait revenir au Jour des Morts puisque, s'il faut en croire le pudibond Villarroel, hommes et femmes s'entassaient sous les arcades sous prétexte de voir les offrandes alors qu'en réalité :

Esta concurrencia, no es otra cosa que una permitida escuela de liviandad, donde con achaque de la confusion y multitud, se alarga a todo género de licencias indebidas, siendo continuos los pellizcos, los manoseos, los estrujones, y otros precursores de la lacivia... ${ }^{2}$.

Les cimetières bénéficiaient, eux aussi de la bruyante bonne humeur qui entourait les actes de la foi puisque la coutume s'était introduite d'enterrer les défunts à grand renfort de trompettes par les ancêtres des mariachis, en quelque sorte ${ }^{3}$.

Pour en revenir aux affrontements créoles-gachupines, la polémique n'épargna pas les deux vierges majeures : la vierge espagnole, Nuestra Señora de los Remedios, d'un côté et, de l'autre, la célébrité locale, la vierge indigène, Nuestra Señora de Guadalupe. Entre autres choses, ce qui échauffait la bile des créoles, c'était qu’à chaque époque de sécheresse, quand il s'agissait d'organiser des processions pour implorer la pluie, qui sortait-on de sa niche ? Nuestra Señora de los Remedios. Et les créoles de maugréer ce qui, d'après Humboldt, était devenu un proverbe : " i hasta el agua nos ha de venir de la Gachupina! ».

Que la Vierge de Guadalupe ait servi d'étendard au curé Hidalgo ne fut pas de nature à arranger les choses, bien sûr, mais même des royalistes convaincus s'émurent d'apprendre que dans leurs troupes, des soldats, pratiquant la polémique à son niveau le plus élémentaire, celui de l'injure pure et simple, la traitaient de " puta " et " alcahueta » (ce qui me fait indéfectiblement

1. "¿Qué les parecerá en un acto tan serio, el ver ir vendiendo en la procesion y por toda la carrera a los de la infima plebe, matracas, judas, dulces, frutas, aguas, y demas comestibles con gritos y voces descompasadas, en unos dias de jueves y viernes santos, que deben ser los de mas compunsion y veneracion de los cristianos?" Enfermedades políticas que padece la capital de la $N$. España en casi todos los cuerpos de que se compone y remedios que se deben aplicar para su curacion, México, 1831, p. 77.

2. Ibid., p. 76 .

3. «S.D. De todas materias ha hablado hasta el dia, menos de una mala introduccion que hay en los entierros, de tanta trompeta y otros instrumentos bélicos, que mas parecen los entierros fandangos o batalla de teatro, que seriedad y sentimiento. " Lettre au Diario de México, $\mathrm{n}^{\circ} 131$, 8 de febrero de 1806.

4. Alejandro de Humboldt, Ensayo político sobre el Reino de la Nueva España, México, Editorial Porrúa, 1966, p. 144. 
penser à cet aumônier espagnol de mon enfance qui venait nous enseigner un catéchisme ponctué d'innombrables "; Me cago en Dios!"). De leur côté, pour attiser la haine contre les Espagnols et toujours pendant le conflit, les insurgés faisaient circuler l'historiette suivante : Que Nra Sra de Guadalupe habia dicho a Nra Sra de los Ángeles : mira niña lo que han hecho: Mariquita de los Remedios, siendo más fea que nosotras, más Chiquita y cacarañada, sólo por ser Gachupina, le hacen caso ; y no de nosotras porque somos Americanas " ${ }^{5}$. Comme le constata un adversaire des insurgés, la guerre d'Indépendance se transforma en véritable guerre des deux Vierges. En effet, écrivait-il, muchas veces se ha dicho entre los rebeldes autorizados, que la imagen de Guadalupe está en guerra con le de los Remedios a quien dan el nombre de "la gachupina ".

\section{LAS DÉCIMAS SON BUENAS PARA QUEJAS}

Comme l'a fait remarquer un linguiste allemand, " polémiquer est plus qu'exercer une simple critique. Cela implique toujours que celui qui polémique reste toujours en deçà d'une norme de bienséance et d'objectivité " ${ }^{7}$. Et, en effet, les débordements de langage étant inhérents aux querelles, celle qui opposa les frères ennemis n'en fut évidemment pas exempte. Pour nous en débarrasser je commencerai par évoquer l'exemple le plus tardif qui date de 1813, en pleine guerre d'Indépendance. Le 13 avril de cette année-là, une espinela placardée sur les murs de Querétaro exposa aux passants une profession de foi plus marquée par la conviction que par la bienséance. En effet, même si le respect scrupuleux de la structure poétique des décimas qui, comme on le sait depuis Lope, son buenas para quejas, même si l'usage énergisant de l'anaphore pour traduire la détermination du poète témoignaient d'une maîtrise parfaite de cette forme poétique dans sa rigueur classique (abba/ac/cddc), une certaine rudesse lexicale pouvait être l'objet de réticences. $\mathrm{Si}$, comme on a pu l'écrire, un des traits du discours polémique est que "sa visée, perlocutoire, est une disqualification de sa "cible", c'est-àdire du protagoniste avec lequel on polémique ", il sera difficile de trouver plus polémique que le petit poème qui suit :

5. Informe de fray Simón de Mora a la Inquisición de 22 de febrero de 1811, Hernández y Dávalos, Colección de documentos para la historia de la guerra de Independencia, tomo I, p. 110.

6. Ibid., tome III, p. 806, D. Fermín de Reygadas, El Aristarco, n 18.

7. Artur Greive, "Comment fonctionne la polémique ? " in Roellenbleck, Geor. Le Discours polémique : aspects théoriques et interprétations. 1985. Colloque de Cologne, mai 1978, p. 20. 


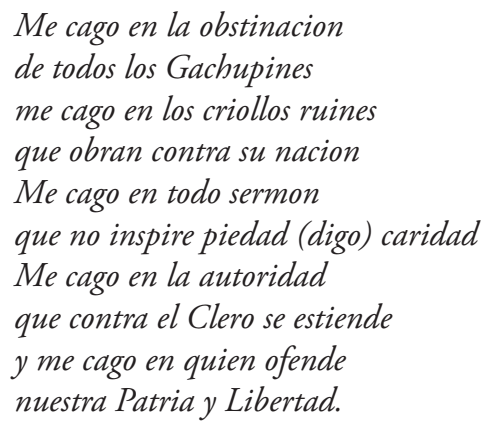

Cette vigoureuse vitupération défécatoire s'inscrivait, en fait, dans un précédent récent d'utilisation de la décima à des fins imprécatoires. Un lustre auparavant, dans la capitale, issue de la main des commerçants espagnols du Parián, le quartier des boutiques, une espinela parfaite elle aussi dans sa forme avait été affichée pour l'illustration de la clientèle. Elle exposait les résultats d'une prétendue recherche étymologique qui puisait dans deux langues différentes afin de faire toute la lumière sur le mystère originel du mot identifiant les frères mexicains. Bien entendu, à travers le mot, c'est l'être même du créole, sa nature profonde qu'il s'agissait de dévoiler. La fantaisie linguistique y rivalisait avec les mauvaises intentions et la volonté de disqualification par ridiculisation y était indiscutable. $\mathrm{Si}$, comme on a pu l'écrire, "le discours polémique vise à tromper l'attente de l'interlocuteur ", le poème répondait on ne peut mieux aux exigences du genre. Qu'on en juge :

En la lengua portuguesa
al ojo le llaman CRI,
y aquel que pronuncia así
aquesta lengua profesa.
En la nacion holandesa
OLLO le llaman al culo
y asi con gran disimulo,
juntando el CRI con el OLLO,
lo mismo es decir CRIOLLO
que decir OJO DE CULO.

En revanche, l'autre objectif fixé au discours polémique, celui de réduire au silence l'adversaire, ne fut pas atteint. En effet, si cette attention alla sans doute droit au cœur des créoles, pour ne pas être en reste, ils n'eurent de cesse de fixer leur réponse, au même endroit et sous la forme, eux aussi, d'une décima non moins réglementaire que celle qui avait été composée à 
leur encontre et non moins débordante d'inventivité linguistique. Enrobant hardiment l'injure d'érudition fantaisiste et, rendant métissage étymologique pour métissage étymologique, elle répliquait :

GACHU en arábigo hablar
es en castellano mula:
PIN la Guinea articula
y en su lengua dice DAR,
de donde vengo a sacar
que este nombre GACHUPIN
es un muladar sin fin,
donde el criollo siendo culo
bien puede sin disimulo
cagar en cosa tan ruin.

Si la joute poétique se poursuivit, elle n'a pas laissé de traces à ma connaissance. Grâce à ces deux cas, "nous voyons à présent beaucoup mieux les raisons pour lesquelles, dans la polémique, la forme artistique du discours, pour reprendre l'allusion du Meyer, que Jakobson qualifiait de forme poétique, joue un rôle important $»^{8}$.

\section{Tecomates et agachupinados}

Comment distinguait-on un créole d'un gachupin ? Essentiellement à leur manière de prononcer leur langue commune et cela donna matière à un débat, entre créoles, cette fois, qui occupa pendant des années les colonnes du Diario de México, ce quotidien né en octobre 1805 et qui fut le vecteur de toutes les polémiques, alimentées par des lettres de lecteurs, acheminées par la poste ou, dans la capitale, déposées dans des boîtes prévues à cet effet, les buzones, équivalents mexicains des bouches de fer imaginées en France, en 1789, par l'abbé Fauchet, fondateur d'un club social et du journal du même nom : La bouche de fer.

Beaucoup plus mesurée et bienséante que la précédente, la polémique allait opposer, pendant cinq ans, du numéro 4 du journal au numéro 1439, à travers 28 lettres au journal, les tenants d'une prononciation de l'espagnol à la castillane et les défenseurs de la prononciation créole. Pour une fois, le règlement de comptes s'effectua strictement entre créoles, même si le juge-

8. Ibid., p. 23. L'allusion au Meyer renvoie au début de l'article : "Pour le Meyers Konversationlexicon de 1890, la polémique n'est pas dispute en soi, mais 'l'art de mener la dispute' ", p. 18. 
ment porté par les Espagnols sur la manière de parler des créoles constituait un élément important du problème. Le débat qui, soit dit en passant, apporte des informations intéressantes sur les pratiques orales de l'époque, portait sur la prononciation des $c, z$ et $l l$. Seule une minorité de créoles s'appliquait à prononcer correctement et se faisait traiter de " agachupinados " par les autres qui, en contrepartie, étaient qualifiés de " tecomates ", c'est-à-dire de gourdes, par leurs compatriotes. L'orthographe, cause pour certains, effet pour d'autres de la confusion des consonnes, servait d'illustration supplémentaire aux ravages dénoncés de cette mauvaise pratique.

Dans un premier temps, les défenseurs de la norme castillane furent seuls à s'exprimer : el Criollo Mexicano, el Escuelero de Tierradentro, L. P., Criollo Pueblano, affligés ou indignés, firent entendre un chœur unanime de réprobation de l'ignorance têtue de la plupart des créoles, "la ingratitud, injusticia, ignorancia y torpeza de nuestros paisanos " manifestes, y compris dans les plus hautes sphères de l'aristocratie locale. Le maître d'école le fit sous la forme d'une anecdote en retranscrivant une discussion sur le sujet avec un de ses élèves qui l'avait traité de "pedante ridículo ". Le Criollo Otomí, pour sa part, choisit de décrire les affres de l'ignorant au moment de devoir rédiger une lettre destinée à un correspondant pour rendre le drame plus tangible, composant un petit tableau costumbrista:

\begin{abstract}
...que quando se le ha ofrecido escribir alguna carta a sujeto que lo entiende, se pone primero a discurrir, ¿como deberá ser? y supongamos caza o casa, y despues de estar suspenso un gran rato en cosa que duda (que son muchas) sube el dedo a la boca, eleva los ojos a las vigas, se rasca velozmente la cabeza, y al fin lo yerra: digo que lo yerra, por que no contento, por el qué dirán, consulta, pregunta, y aun enseña su carta a algún amigo castellano viejo : éste se la corrije, y si tiene tiempo, la traslada, y si no, en todas aquellas partes en donde ha puesto z por $c, o c$ por z, o z por s, o s por z, o s por c, o y por ll, o ll por y, les deja caer un borroncito para que duden lo que es; ya V. puede considerar, que tal irá la carta, pues aún él mismo las mas veces no la entiende 9 .
\end{abstract}

Un autre intervenant anonyme, tout en se défendant de vouloir "zaherir a nuestros paisanos ", se fit un plaisir d'ajouter au tableau quelques éléments omis par ses prédécesseurs, comme le déplacement de l'accentuation qui transforme " reí " en " rey ", " cai " en " cái » avec pour effet, par exemple de réduire les trois mots "oya ", "olla " et "oía " à un seul, " oya ", en l'occurrence. Sans se priver d'ironiser, au passage, aux dépens de l'Église, en

9. Diario de México, núm. 173, 22 de marzo de 1806. 
constatant que « Es bien conocido por acá Isáyas de muchos predicadores ${ }^{10}$. Et pour faire bonne mesure et bien marquer son intention de ne pas mortifier ses congénères d'ajouter que la musique ne valait guère mieux que les paroles puisque, expliquait-il :

Agrégase el que nuestros paisanos, por lo común, pronuncian todas las voces en un modo arrastrado, oprimido, estrujado, confuso, con un sonido ingrato de suerte qua aun las letras que articulan, no se perciben con bastante claridad, y distincion.

Six mois et une douzaine d'articles plus ou moins offensants après, les créoles tecomates relevèrent enfin la tête et se lancèrent dans la défense de leur parler propre par la plume d'un des leurs, un créole de la Villa de Santander qui signa modestement El Payo Tierradentreño. Le soi-disant plouc fit remarquer que la manière de prononcer des créoles comme celle des Andalous ou d'autres péninsulaires n'empêchait nullement à la langue de remplir sa destination première, celle de se faire comprendre. Pour lui, l'attribution d'un son à une lettre relevait de l'arbitraire pur et simple et vouloir que cela se perpétue "mas es capricho que justicia " ${ }^{11}$. Et pour la mélodie, il affirmait "ser muy dulce y clara la pronunciación del criollo con respecto al Europeo... al menos a mi me yere mas suave el organo del oido ".

Aussitôt, encouragé selon ses dires mêmes par cette première riposte, le relais fut pris par El Criollo refinado, bien décidé à rendre coup pour coup à tous ceux qui avaient exprimé leur honte devant les massacres consonantiques perpétrés devant les Européens. La honte, le nouvel intervenant dit l'avoir ressentie devant les créoles qui voulant imiter la prononciation des péninsulaires commettaient d'énormes bévues et se couvraient d'un ridicule qui rejaillissait sur tous les créoles ${ }^{12}$. Surtout, il dénonça avec vigueur la trahison à la cause des créoles que constituaient les comportements de ces méprisables adulateurs du modèle étranger et la polémique en vint à prendre un tour plus politique. De ceux qu'il accusait de refuser la tradition et de bouleverser ce qu'il appelait "la constitucion nacional de nuestro idioma » en forçant leur nature dans l'imitation, il déclara :

10. Ibid., n² 206, 20 avril 1806.

11. Ibid., n 236, 24 de mayo de 1806.

12. "...de algunos años a esta parte observo, que muchos de nuestros paisanos, especialmente en esta Capital de México, haciendose pedantes, y ridiculos en la materia, quieren usar de la pronunciación gachupina, particularmente en la ll ; y ya sea porque no estan muy acostumbrados, o por que ignoran las letras con que se distinguen los vocablos, sueltan tales disparates, que la cara se me ha llenado de rubor al oirlos, algunas veces en concurrencia de Europeos, decir la chirimolla, y llo, alludeme V., la meza, el paztel. ». Ibid., n 291, 18 de julio de 1806. 
Censuran ellos mismos nuestra pronunciacion criolla, tratandonos a los que la acostumbramos con la expresion de tecomates. ¡No ven pues, que ellos mismos se envilecen con esto! Dan a entender que es vil, y despreciable el criollismo; y no pudiendo desnudarse de la qualidad de criollos, como que se avergüenzan de serlo, se despojan del mas perceptible distintivo qual es el de la pronunciacion.

Au Criollo refinado répondit un Criollo no refinado par une longue réfutation et le vœu, in fine, qu'il cesse d'encombrer le Diario de ses contributions "nocivas a la cultura Americana " ${ }^{13}$. Le Payo tierradentreño, lui, eut droit à une tentative de ridiculisation, plus ou moins réussie, signée Sanson Carrasco, parce qu'écrite en style pseudo quichottesque, avec abondance d'archaïsmes et toute entière, ou presque, construite sur l'ironie et la dérision. Ainsi, cette apostrophe, par exemple :

$Y$ tu, o luz y norte de la andante literatura, que qual otro Apolo venistedes desde el Olimpo de Santander a ser maestro de pastores y gañanes, Numen sagrado de los poetas de rancho, y de baratillo, juntad ahora las fregonas, y verduleras, y forma tu parnaso ${ }^{14}$.

Le malheureux payo est transformé en don Quichotte libérant les enfants de la sujétion à l'étude et aux précepteurs, affrontant les géants Horace et Quintilien, bouchant ses oreilles aux paroles enchanteresses des Homère, Virgile et autres et prônant la liberté qui naît de l'ignorance des règles ${ }^{15}$. La cruauté finale consista à reprendre les fautes d'orthographe du texte du payo, que le rédacteur du journal, partisan de la prononciation à la castillane, s'était refusé à corriger, comme le lui demandait l'auteur :

Solo nos resta aclarar el engorro de esa maldita ce y zeta, pues no todos sabrán usarla, u omitirla con tanto acierto como vos la usastedes, en las voces interezantes, prenza, paizanos, y omitistedes en las produsidas, complasido, disen, $y$ demas; ni todos tienen tan bien templados sus oidos, que sepan quando suena dulce, y quando aspero: quando debe decirse pronunciacion y quando pronunsiacion, etc.

13. Ibid.., n 429, 3 de diciembre de 1806.

14. Ibid., $\mathrm{n}^{\circ} 303,30$ de julio de 1806.

15. «Sostened con buen talante la simpleza, o simplicidad de vuestro sistema, de que las reglas de bien hablar y escribir, se reducen a darse a entender cada uno de la manera buena o mala, que ha aprendido, sin cuidar del sonido de las letras, del significado de las palabras, ni menos del artificio de las frases y figuras. Haced ver, que para hablar, y escribir es necedad sugetarse a las reglas que prescriben la gramatica y esas otras faramallas, que llaman retórica y eloqüencia... " Ibid. 
Je terminerai par une lettre signée Pepita Gamunz qui eut le don d'exaspérer le rédacteur du Diario, défenseur de l'orthodoxie castillane. La pseudo Pepita, un homme en réalité comme le dénonça hargneusement le journaliste dans une note ${ }^{16}$, revenait sur le thème du ridicule qu'il y avait à vouloir prononcer à l'espagnole, en décrivant la confusion de tout un groupe de créoles devant les pataquès $\mathrm{d}$ 'une jeune femme, auparavant vive, naturelle et enjouée mais qui, influencée par les remontrances publiées dans le Diario, avait tout perdu et sombré dans le ridicule jusqu'à devenir « una gachupina muy refinada ${ }^{17}$. C'est le journaliste lui-même qui nous fournit les raisons de sa fureur en dévoilant que la cible des moqueries n'était pas un être de fiction créé pour les besoins de la polémique, mais une personne bien réelle, l'actrice Luz Vallecilllo, première dame du théâtre de Mexico, dont le pauvre journaliste, tout en prenant ardemment sa défense reconnaissait implicitement qu'elle n'était pas exempte des travers dénoncés ${ }^{18}$.

Toujours dans le domaine du langage, mais un peu spéciale et beaucoup plus expéditive puisqu'une réplique suffit à y mettre fin, il y eut aussi une brève polémique soulevée par un lecteur autour du langage du sifflet. Dans une lettre, celui qui signait imprudemment El Bascongado, dénonçait l'existence à Mexico d'un idiome infâme, pratiqué par le peuple "soez " pour communiquer à coups de sifflet et préparer des mauvais coups de toute sorte, depuis les rencontres honteuses entre les deux sexes, jusqu'aux meurtres, au vol, au jeu et à la fornication. Il exigeait qu'il soit mis fin à cette barbarie unique au monde, comme il pouvait s'en porter garant en homme qui " ha corrido las quatro partes del globo, y solo en este continente ha notado

16. "Debemos advertir que la letra de la carta no indica ser de mujer. La Señora Pepita se valió sin duda de su hermano el Bachiller o de algún gramátiquillo de tres al cuarto, que aunque indica que oyó algunas reglas de ortografía, no las sabe y las quebranta en la misma carta. "Ibid., $\mathrm{n}^{\circ}$ 707, 6 de septiembre de 1807.

17. "ique risa daría a V. aunque sea V. mas serio que un Quixote, al oirla : al pazar por la plasa aller de mañana, la encontré con aquellia paziensia que acostumbra: (...) D.N. que estaba presente, se puso como unas escarlatas, y mi madre dió la vuelta con mucho disimulo : padezco tales mortificaciones, que sea por Dios, por que en efecto no podrá remediar aquella aspereza, y cierto refinamiento ridiculo, que es capaz de chocar y lastimar a los oídos de un herrero; no parece sino que pronuncia con dos zz corazzon; y que despues de la ll siempre pone una $\mathrm{i}$, para decirnos ellia, halliazgo, etc y en cuanto a la c, y s, de esto no se hable». Ibid., n ${ }^{\circ} 706,5$ de septiembre de 1807.

18. "...habiendo estado un año en la Havana, volvió a presentarse aquí luciendo su papel con mejor aire, y mas expresiva en la accion, y pronunciando bien la $z$, la $c$, y la ll, no siendo de extrañar que se le escape una, $u$ otra, por que un año no es bastante tiempo para destruir enteramente un hábito de muchos». Ibid., nº 707, 6 de septiembre de 1807. 
el uso de este lenguaje que es propio de cabreros y pastores en las montañas y matorrales" ${ }^{19}$.

Le pauvre Basque ne soupçonnait certainement pas ni l'agressivité ni la nature de la réponse que son indignation allait lui attirer. Les léperos du bas peuple n'étaient pas l'objet d'une sympathie particulière de la part des créoles qui n'étaient pas les derniers à les mépriser ou, dans le meilleur des cas, à s'en méfier. Mais, c'était leurs léperos et c'est à eux qu'il revenait de les vilipender, le cas échéant, mais surtout pas à un gachupin, basque de surcroît et qui assimilait Mexico à une contrée sauvage et barbare. La réponse fut donc largement à la hauteur de l'attaque et elle s'articula en deux parties inégales : la première, la moins originale et la plus grossièrement facile, fut l'insulte, l'auteur de la réfutation se mettant en scène comme un combattant à cheval, descendu dans l'arène pour placer quelques piques sur l'échine du taueau basque, en jouant sur le mot corrido pour transformer en corrida la polémique ${ }^{20}$.

La deuxième partie était à la fois la plus longue et la plus originale, puisqu'elle consista à transformer l'érudition en arme de la polémique. Il y a fort à parier, d'ailleurs, que le temps respectable mis à rédiger une réponse ait été mis à profit pour rasssembler de la documentation, puisque c'est une véritable histoire du sifflet à travers les âges qui fut déversée sur le Bascongado pour lui jeter à la face son ignorance et dévaloriser ainsi tout son discours. L'argumentation commença contre l'affirmation du Basque comme quoi il n'y a qu'à Mexico que les sifflets servent à accomplir des actes mauvais : c'est de la Bible que jaillit la réfutation et c'est Dieu lui-même, selon le prophète Isaïe qui en avait donné l'exemple, en sifflant les mouches d'Égypte et les abeilles d'Assyrie pour qu'elles viennent piquer les Juifs ${ }^{21}$. Suivaient les exemples d'Ovide dans les Métamorphoses qui parle des trois coups de sifflet d'Esculape pour dire aux Romains qu'il les favorisait, de Tite Live qui

19. Diario... op. cit., $\mathrm{n}^{\circ} 40,9$ de noviembre de 1805.

20. "...vengo hoy en mi penco a poner un par de varas al Bascongado del diario núm. Tomo la garrocha, y salgole al encuentro. ; Mosca! y que cerviguillo que tiene! Parece marrajo. Ya se vé, como que es toro corrido por las quatro partes del Mundo. Sin embargo; doyle un silvido... parece que se ha avivado... ; ola hermano ! ¿ con que solo en México ha oído silvar? Pues escucheme quatro palabras, que serán otras tantas garrochas que le plante. "Diario... op. cit., $\mathrm{n}^{\circ} 68,7$ de diciembre de 1805 .

21. "Isaías, 7, 18. Y acontecerá que aquel día silbará Jehová a la mosca que está en el fin de los ríos de Egipto, y a la abeja que está en la tierra de Asiria; 19, y vendrán y acamparán todos en los valles desiertos, y en las cavernas de las piedras, y en todos los zarzales, y en todas las matas. Mensaje de Isaías a Acaz. " La version donné dans le Diario est un peu différente : "que dara Dios un silvido, y acudirán al instante las moscas de Egipto y las avejas de Asiria, y vendran a picar a los Judios". 
raconte la conquête de Tarente par Hannibal grâce à un coup de sifflet de Philomène pour que les gardes ouvrent les portes, d'Apulée qui rapporte qu'une veuve proposa à son beau-frère de venir la retrouver de nuit " y al primer chiflido que dieres te abrirá la puerta mi criada », de Quevedo dans le Buscón : du dictionnaire de Trévoux qui explique qu'en France, siffler est le langage des laquais et des gens de bas étage. D'où le retournement complet de la pique finale qui place le Mexicain des bas-fonds au pinacle, au pinacle des siffleurs bien entendu :

...no nos venga otra vez el seor Bascongado a hacer del melindroso, del culto, y del viajante. Por que lo mas que podrá concluir, si se empeña, es que la plebe de México ha dado la última perfeccional idioma chiflesco, en que fueron balbucientes los Griegos, Romanos, Africanos y Españoles de los tiempos de antaño.

\section{LE PROYECTISMO, TERRITOIRE DE PRÉDILECTION DE LA POLÉMIQUE}

Au XVIII', tant en Espagne qu'en Amérique, le proyectista a pris la suite des arbitristas des deux siècles précédents Produit du rationalisme, le projet se distingue par ses intentions utilitaires et, souvent, l'utilisation de la statistique. Sa définition la plus complète est celle proposée par l'historien espagnol José Muñoz Pérez pour qui le projet :

intenta solucionar una cuestión concreta, que puede ser de las más diferentes ramas de la administración, de la sociedad o de la economía del pais, partiendo de una base de conocimiento, teniendo en cuenta la serie de conexiones que lo enlazan con el resto de la problemática general y graduando sus posibilidades de aplicación en una serie de etapas ${ }^{22}$.

Dans le Prospecto exposant le programme du Diario de México, son fondateur, Jacobo de Villaurrutia se présentait comme El proyectista et promettait, à ce titre, de proposer ses propres projets au public, mais aussi d'accueillir dans ses colonnes tous ceux qui lui seraient transmis. La promesse fut tenue et c'est de quelques uns de ces projets qui donnèrent lieu à polémique que je vais parler maintenant.

Ainsi, en 1807, un article inttulé Economía rural rapporte un dialogue qui aurait eu lieu entre le signataire, El Melancólico, pseudonyme d'un des rédacteurs du Diario, J. W. Barquera et un certain Vizcaino nommé Julio

22. Muñoz Pérez, José. "Los proyectos sobre España e Indias en el siglo XVIII : el proyectismo como género ». Revista de Estudios Políticos, mayo-junio 1955, nº 81, p. 169-195. 
César. Ce dernier, comparant le rendement au travail des paysans espagnols et de leurs homologues indiens concluait que la différence qu'il trouvait, en faveur de ses compatriotes, était due à leur force physique supérieure, celleci, à son tour, étant directement fonction de leur mode alimentaire. Partant de là, il se faisait fort de tripler le rendement des Indiens en modifiant leur système nutritionnel. Il proposait que dans les haciendas, le propriétaire prélève deux réaux par semaine sur le salaire de ses journaliers indiens et se charge de les nourrir en leur fournissant "una grande olla de carne, otra de frixoles, y otra de habas » et en remplaçant la tortilla ancestrale par du pain. Ces repas pris en commun auraient l'avantage supplémentaire de permettre aux intéressés de "perfeccionarse en el idioma " ${ }^{23}$. Le corollaire profond du changement ainsi proposé n'était rien moins qu'une tentative d'intégration de l'Indien à la société d'origine européenne par une sorte de communauté, sinon d'identité, physiologique produite par l'assimilation des mêmes aliments.

Le premier à répondre ne put résister au plaisir d'hyperboliser ironiquement à partir du nom du proyectista en déclarant :

....yo aseguro desde ahora para entonces al Vizcaino Julio César, que aunque viniera el Romano con el exército con que derrotó a Pompeyo en los campos de Farsalia, no haría salir de su paso a los trabajadores del campo de esta tierra, mucho menos despues de haberlos atracado, como intenta, de carne, habas, y frijoles. El hombre es tanto mas feliz, cuantas menos necesidades conoce ${ }^{24}$.

L'essentiel de la longue réfutation qui suivit tournait principalement autour d'une affirmation : ce qui manquait aux Indiens, ce n'était pas la robustesse mais l'ardeur au travail, une activité à laquelle ils ne se prêtaient que contraints ou forcés, soit par autrui, soit par leurs propres nécessités. Les lettres successives abonderont dans le même sens, en mettant en garde les ingénus qui seraient tentés de mettre en pratique ces funestes conseils et la polémique s'acheva au bout de trois ou quatre articles par la déroute du projet gastronomique. Mais, elle a le mérite de présenter une originalité dans la manière de conduire la controverse. Un des adversaires du projet, en effet, au lieu de dérouler comme les autres son argumentation, innova en faisant intervenir dans le débat ceux-là même qui étaient l'objet de la sollicitude alimentaire, à savoir les Indiens. C'est à l'un d'eux, qu'est prêté un discours qui, en même temps qu'une réfutation, alliant comme c'est souvent

23. Diario... op. cit., $\mathrm{n}^{\circ} 709,8$ de septiembre de 1809 .

24. Diario... op. cit., $\mathrm{n}^{\circ}$ 805, 13 de diciembre de 1807. 


\section{AUTOUR DU DISCOURS POLÉMIQUE EN NOUVELLE ESPAGNE}

le cas, le comique à la polémique, propose une pittoresque parodie de l'espagnol parlé par les Indiens, avec bouleversements syntaxiques et autres caractéristiques, ce savoureux échantillon ajoutant une page de plus aux exemples de différents parlers que l'on peut trouver dans le Diario (parler des payos, "indiotismos " 25 , etc.). Ici, il s'agit d'un berger indien qui, ayant entendu parler de la proposition de Julio César el Vizcaíno, se met en route pour se faire engager chez lui, mais rencontre en chemin son grand-père qui lui tient un long discours dissuasif et si savoureusement imagé qu'il m'est impossible de ne pas en citer de larges extraits :

no cres hijo tal cosa, ese es un grandisma mentira, y el escribidor, o no tiene su hacienda, o está peliado con su dinero: ¿no ves que buen coleada le da el Ranchro? estátelo en to xacal, comiendo tortiyas y chile y bebiendo to atole que es tu costumbre y no vayas por ese tierra a padescas lo que tu burro, que cundo comías rastrojo buen borriquito, y desde que probaste el cebada, ni cargas cantaros ni petate, ni tienes pelo ni le quedó su cola: mira hijo, cuando nació ese encina viejo del tetel, ya yo y otros del pueblo sirviamos vara de topile de Santa Iglesia, y siempre nuestros diente cabal, sin que dueles, ni que te pugras, blanco como el nieve nuestro boca, el melenas y copete negro, juertes, juertes en trabajo de monte, de campo, de pastorias, y baqueria echamos lazo al toro brabo, y pones yugo u solo, por que atole, y chile con pulque da muchos juerzas. En cuanto comes pan y carne, te vuelves floxo, y rascas barriga, y te enfermas, y te canta tecolote, $y$ te mueres, $y$ te entierran en sepultura jondo ${ }^{26}$.

25. Par exemple, un article intitulé Idioma del lenguaje payuno, une lettre présentée comme " una coleccion completa de las palabras, y frases de la gente poco culta de las provincias, y por que no carece de gracia en la sátira, y el ridiculo " et dont voici un court extrait : " hagas cuenta quere disir que no es lo que queren que sea y sinó diganlo estos dos perversos payunos que nos jinos yo yuna saparrastrosaunocasion que nos peliamos le dije y o ella

Si e ia no jueren faldada

o si jueras calsonudo

por que me dixo cornudo

le dieruna bofetada

llentonses él ya terciandose el paño por debajo del sovaco derecho me respondió,

Haga cuentell muy perico

de que yo treigo calsones

bera como a moxicones

pedasos li agolosico (pedazos le hago el hocico)"

Diario..., op. cit., n ${ }^{\circ} 445,19$ de diciembre de 1806.

26. Diario..., op. cit., 26 de diciembre de 1807. 


\section{VÊTIR CEUX QUI SONT NUS : LE PROTOTYPE DU PROJET}

Une des particularités de la société mexicaine qui fit couler beaucoup d'encre et sans doute aussi beaucoup de salive fut la quantité énorme de vanu-pieds plus que sommairement vêtus qui peuplaient les rues de la capitale. Connus sous le nom de zaragates, comparés par Humboldt aux lazzaroni napolitains, ces désœuvrés pacifiques étaient au nombre de 30 ou 40000 d'après le savant allemand, mais ils n'étaient pas les seuls à ne porter que quelques guenilles sur le corps et une grande partie des travailleurs n'étaient pas mieux lotis. Les ouvriers boulangers, par exemple, n'avaient la plupart du temps qu'une manta pour s'abriter à deux quand ils allaient à la messe du matin, de sorte que le moindre faux-mouvement apportait sur leur intimité des révélations soudaines de nature à effaroucher les catholiques matinaux. Moins coutumier mais plus spectaculaire avait été l'incident survenu lors de la grand messe dans la cathédrale, quand au moment où il se tournait vers les fidèles, l'Indien qui servait la messe perdit sa tilma (tunique), son seul rempart contre la nudité et, pour reprendre les termes de Villarroel qui relate l'anecdote, "se quedó en cueros vivos, a vista de un gran concurso que la estaba oyendo en la Catedral $»^{27}$.

Cette situation avait toujours été dénoncée comme une tare sociale, une atteinte à la réputation de la capitale et la pression publique avait même débouché sur un édit du vice-roi Azanza enjoignant aux court-vêtus de remédier dare-dare au désordre de leur toilette sous peine de se retouver entre quatre murs. Il y eut quand même une voix pour s'élever contre cette contrainte infligée aux misérables - et qui n'eut, d'ailleurs aucun effet véritable - et pour dénoncer une autre sorte de nudité, celle que la mode favorisait chez les dames. José Manuel Sartorio, un prêtre poète, "ramplón, aniñado, humilde", publia ce sonnet vengeur "Sobre el bando que condenó a cárcel a los pobres desnudos » où sa pudeur offensée s'exprimait dans une exclamation où l'indignation semble le disputer comiquement à l'admiration :

Una manta a su cuerpo trae pegada, y tal vez nada más, la pobre gente, mas no ofende al pudor, pues finalmente es su tápalotodo una frazada.

Chupa y calzones lleva una alindada currutaca persona: es evidente;

27. Enfermedades..., op. cit., p. 79. 
mas los bultos descubre impuramente de partes y trasero. ¡Ay, que no es nada!

\author{
No obstante, la celosa policía \\ perdona a ese tapado descubierto \\ que más bien la sentencia merecía, \\ y condena al desnudo, aunque cubierto, \\ ¿Esto por qué será? Juro a fe mía, \\ que es porque el pobre siempre hiede a muerte.
}

Le premier projet publié dans le Diario, dès son $n^{\circ} 6$, prit le contre-pied absolu de toutes les opinions antérieures en déclarant d'emblée que la masse de ces miséreux constituait une richesse sans égale pour la pays ou, tout au moins une richesse en puissance. Le paradoxe de départ s'expliquait par le fait que les vêtir et les chausser entrainerait un développement sans précédent de l'artisanat des tailleurs et des cordonniers et, en amont, des ateliers de tissage et des filatures comme des cultures textiles et de l'élevage pour le cuir, la laine, etc. sans oublier la métallurgie mobilisée pour la fabrication des outils nécessaires à cette explosion des activités ${ }^{28}$. La polémique se déchaîna aussitôt, mais ce qui est remarquable c'est qu' elle ne porta pas sur le caractère utopique ou non du prodigieux enchaînement de causes et d'effets qui devait amener à la prospérité à une vitesse foudroyante. Apparemment, la mécanique implacable de la démonstration découragea toute entreprise de contestation dans ce domaine. En d'autres termes, la dimension économique du projet ne fut pas l'objet d'une réfutation et c'est sur ses incidences sociales supposées que les hostilités se déclenchèrent. Six jours après la publication du projet, les deux premières lettres de lecteurs entamèrent le débat. La première, très brève, se contentait d'être injurieuse pour l'auteur, coupable de s'intéresser à la lie du peuple ${ }^{29}$. Beaucoup plus intéressante, la deuxième lettre utilisait le procédé signalé plus haut, l'auteur feignant d'être un des supposés bénéficiaires du projet pour prôner le maintien du statu quo, avec une gouaille réjouissante, destinée à mettre les rieurs de son côté :

28. Sur le fond du problème, je me permets de renvoyer à mon article «Analyse sociale et philosophie pratique : autour de la notion de besoin en Nouvelle Espagne à la veille de l'Indépendance ", in Structures et cultures des sociétés ibéro-américaines, Bordeaux, Éditions du CNRS, 1990, p. 126-148.

29. "Señor proyectista: Al primer tapón zurrapas. Quien había de créer que despues de tanta fanfarria como gasta U. en su carta preparatoria, precursor o preliminar, habia de empezar sus proyectos por los enmantados, por la ez (sic) del pueblo, de quien nadie hace caso? V. debe ser de la calidad de las moscas que siempre van a pararse en la inmundicia de las mataduras. "Diario..., op. cit., $\mathrm{n}^{\circ} 12,12$ de octubre de 1805. 
Señor Diarista: Yo soy un encuerado, anoche dormi en el suelo, pero mejor que V. y otros en el colchón. Hoy pienso lavarme muy bien las piernas, y cate $V$. que estreno medias ipara que pues me quiere $V$. meter en la necesidad de comprarlas? (...) El hombre es mas feliz mientras menos necesita ¿para que pues me quiere V. gravar, en lo que he no he menester, solo para lucir discursos exoticos?

El hombre quando se acerca mas al estado natural, es menos dependiente del capricho de sus semejantes, y si yo sin calzones, me rio de los que necesitan tirantes para sujetarlos, y de los que se rien de aquellos, ¿por que se ha de enojar V. por que ando sin ellos? Si la naturaleza, quando me parió mi madre, tuvo por demas proverme de este embarazo ¿que justicia hay para que V. me obligue a él?

Contentese V. con que me cubra con mi manta, no porque la necesite, sino para evitar, que el corazon corrompido del hombre vestido, crea que es desvergüenza dejar de tapar, lo que él por un efecto de su miserable esclavitud.

Conteste V. si es hombre que lo espero con mi cajete en Tumbaburros ${ }^{30}$ mas alegre que una pasqua. El encuerado. S.Z.

Avec une merveilleuse mauvaise foi, on l'aura noté, le pseudo encuerado dénonçait le caractère exotique du discours tout en utilisant le plus exotique des discours, à l'époque, celui du philosophe dont le nom était aussi rigoureusement proscrit que la lecture de son œuvre, mais qui était néanmoins bien connu. Le rédacteur du Diario s'empressa d'ailleurs de commenter la lettre en citant la source de son idéologie avec toutes les précautions d'usage ${ }^{31}$. De toutes les façons, la polémique se poursuivit entre tenants du statu quo et partisans d'une intégration forcée, par le travail, des zaragates et autres encuerados ${ }^{32}$. La réfutation devait venir plus tardivement sous la forme d'un projet subtilement parodique qui, sans en avoir l'air,

30. Ibid., Le cajete était une sorte de chope en terre cuite, d'un demi litre au moins, utilisée dans les pulquerias, Tumbaburros étant l'une des plus connues de Mexico.

31. «(en su carta)... refunde la sustancia de quanto pudo alegar en apoyo de una paradoja un ciudadano de Ginebra, por adquirir gloria, en un discurso premiado en Europa, que segun é (sic) oído decir (por que yo no lo é leído)... Diario... ", op. cit., n 16, 16 de octubre de 1805.

32. Le credo de ces derniers, principalement les rédacteurs du Diario, exprimé à diverses reprises, pouvait se condenser en une phrase : "El hombre nació para la sociedad, y para vivir feliz en ésta, es necsario que trabaje; y no tendrá que trabajar si las necesidades no se aumentan reciprocamente". Diario..., op. cit., $\mathrm{n}^{\circ}$ 805, 13 de diciembre de 1807. 


\section{AUTOUR DU DISCOURS POLÉMIQUE EN NOUVELLE ESPAGNE}

dynamitait tout le fonctionnement usuel des projets et de leurs modalités d'argumentation. C'est avec ce dernier exemple que s'achèvera cette courte visite du paysage polémique.

\section{LA POLÉMIQUE FEUTRÉE}

Il vit le jour en mai 1810, quelques mois avant le début du soulèvement du curé Hidalgo et alors qu'une polémique occupait depuis des mois les colonnes du Diario autour d'un projet intarissable ${ }^{33}$ proposant la création d'une monnaie divisionnaire en cuivre, d'abord pour faciliter les échanges commerciaux de détail, ce qui n'avait rien de choquant, mais aussi et surtout pour faire en sorte que cette monnaie nouvelle permette d'envoyer à la mère patrie tout l'or et l'argent du Mexique, celui des églises, ce qui fit hurler les bons catholiques, mais aussi celui de la monnaie en circulation, ce qui fit vociférer tous les autres. La pelotera qui s'ensuivit, pour reprendre les termes d'un lecteur ${ }^{34}$, s'explique par le contexte où de plus en plus de voix s'élevaient, y compris dans le journal, pour dénoncer que l'or et l'argent produits dans le pays disparaissaient vers la métropole, ce que tout le monde pouvait constater au fait que les pièces en circulation étaient toutes de frappe récente, les plus vieilles ayant dix ans au maximum. Ce sentiment de spoliation alimenta vigoureusement les aspirations à l'indépendance et en 1809, par exemple, furent saisis chez le curé de Huichiapan des billets destinés à être adressés à d'autres curés des environs où il était expliqué que si l'argent cessait de quitter le sol mexicain, au bout de dix ans, même celui qui souhaiterait être pauvre ne pourrait pas y arriver ${ }^{35}$.

33. «Sr. Diarista: Bendita sea Maria Santísima que acabó VM. ya con su moneda de cobre : yo pensé que hablaba Vm. hasta el dia del juicio, y a la verdad que la mitad de lo que dixo (si acaso todo) podia haberse quedado en el almacen de reservas". Diario, op. cit., n 1682, 12 mayo 1810. Zurra a la moneda de cobre. L'auteur du projet était un vieux bethléémite de 75 ans, fray Antonio de San José Muro, gros producteur de projets depuis une trentaine d'années.

34. «Sr Diarista: Al ver la pelotera que se ha suscitado en el Diario contra el autor del proyecto de la moneda de cobre, me compadeci tanto de él, que ansiaba por conocerlo, para advertirle fraternalmente dexase sus pensamientos quiméricos, iguales al condado de Sancho Panza. Mas me compadeci, al ver el bello rasgo de erudicion del Viejo de las novenitas del núm. 1708. Yo decía acá a mis solas, quando un sugeto que cita tantos autores extrangeros reprueba la empresa con precision tan asombrosa, gracia e ironía, es por sin duda un sabio del primer orden, que aun por las uñas brota erudicion, y asi arrímome a su sentir, (...) » Diario..., op. cit., $\mathrm{n}^{\circ} 1722,20 \mathrm{de}$ junio de 1810.

35. "Que de esta America llevan a España diez y ocho millones y algo mas cada año los qe nunca buelben al Reyno y que sino se llevaran este dinero en diez años abria sirculado en el ciento ochenta millones, y qe al respetiba en diez años aunque uno quisiera ser pobre en esta America 
Dès ses premières lignes, le nouveau projet se situait dans le contexte de la controverse autour de la monnaie de cuivre ${ }^{36}$. Bien décidé à régler leur compte aux proyectistas, l'auteur choisit d'avancer masqué, et pas seulement parce qu'il signait, anonymement, si l'on peut dire, d'un simple F. majuscule, mais en se faisant passer pour un ingénu, plein d'admiration devant les propositions des beaux messieurs politicos proyectistas, en particulier celle destinée à fournir le moyen de nourrir tous les affamés du pays

estableciendo fábricas de toda especie de géneros, que aunque nos salieran mas caros, que los que nos vienen de otras partes, al fin se lograría que estos pobres comiesen, el dinero se quedaba en casa, que es lo que importa.

Si j'avais moins peur des mots, je dirais qu'avec ce type de réfutation qui feint de trouver louable ce qu'il convient d'incriminer, on nage en pleine antiparastase. Mais, bien sûr, je ne le dirai pas, quitte à me faire taxer de prétériteur. La reprise, plus bas dans le texte, de la même formule «el dinero se quedaba en casa " laisse fortement à penser que si l'auteur n'est pas espagnol, comme cela semble être le plus probable, il ne peut que faire partie de la fraction conservatrice des créoles qui souhaitaient le moins d'innovations possibles parce que susceptibles de perturber le fragile équilibre social existant. Bien sûr, le développement manufacturier aux vertus de panacée sociale et économique hypocritement encensé renvoie au projet de Villaurrutia de vêtir ceux qui sont nus pour parvenir en un temps record à la prospérité.

Toute la suite du projet va jouer sur le même procédé : présenter avec une pseudo humilité exquise et une délectation savoureuse dans la formulation (" tengo mi miedo de que no me hagan caso, porque, hablando con ingenuidad, yo no tengo estudios, ni he abierto un libro en mi vida, y no ha de tener uno tanto amor propio, que no conozca que es tuerto ") une proposition déclarée née de la contamination par l'esprit proyectista. Comme dans tout bon projet, l'idée de départ en était simple : ici elle consistait à faire prendre un arrêté intimant que toutes les productions locales, blé, maïs, etc. au lieu d'être conduites à destination par le chemin le plus court comme il était coutumier de le faire, soient tenues de parcourir au minimum quatre cents lieues jusqu'à leur point d'arrivée. Par exemple, au lieu de continuer à être amené par barque depuis Chalco, à une trentaine de kilomètres de Mexico, le maïs

no lo abria de poder conseguir . $\mathrm{N}^{\circ}$ 2. Causa del cura de Huichiapan D. Manuel Palacios y el escribano de allí don Manuel Peinvert. A.G.I., México, 5, leg. 1472. Comme on peut le constater, l'orthographe n'était pas le fort du bas clergé.

36. "Sr. Diarista: Sepa Vm. que ayer me hallé en una concurrencia de varios Señores que me dixeron se llamaban politicos proyectistas (no puedo asegurar à Vm. si entre ellos estaba el de la moneda de cobre, porque no lo conozco)». Diario, op. cit., 20 de mayo de 1810. 
devrait d'abord aller jusqu'à Durango et en revenir, c'est-à-dire, parcourir 1800 kilomètres $^{37}$.

Le bénéfice immédiat serait la création d'un nombre d'emplois de muletiers susceptible de fournir un moyen de subsistance à une énorme quantité de désœuvrés, ce genre de métier, contrairement à d'autres, n'étant pas long à apprendre et pouvant être exercé par n'importe quel individu pourvu de bras et de pieds. Comme il se doit, ce projet ne manquait pas de l'attribut réglementaire, à savoir la rapidité magique de l'amlioration introduite : " $y$ asi de un golpe quedaba hecho el beneficio" ".

La démolition discrète par l'absurde se poursuivait avec la mise en ouvre d'un autre trait propre au genre projet : la mécanique des causes et effets, comme pour le vêtement, où l'on partait de la pièce de tissu pour aboutir au développement de la culture du coton, en passant par les fabriques. Ici, cela donnait :

Ademas se fomentaría prodigiosamente la cría de mulas y la de bueyes, si se adoptaba el hacer uso de carros tirados por estos, y esa multitud de tierras que ahora dicen que están vacias, se llenarian en un instante de ganados, y por consiguiente de hombres que los cuidasen.

Yo me lleno de gusto, y creo que a Vm. le sucederá lo mismo, porque al fin todos somos hijos de Adan, al contemplar los caminos todos cubiertos de gentes todos los días, repartiendo dinero por el tránsito, y por consiguiente fomentando los lugares y ranchos que hay en ellos. Entonces si se podría asegurar, que no habría despoblados en el reyno, porque los arrieros los atravesarian por todas partes, y se formarían pueblos, y quando menos mesones que los recibieran. Estos, que ahora son tan mal surtidos y tan incómodos, por ser pocos los que caen, se proveerían de lo necesario, como que tenian la salida y ganancia segura.

Loriginalité de la technique consiste donc ici à faire en sorte que la réfutation prenne l'apparence d'une adhésion aux principes et à la nature même du projet en tant que genre, mais en la chargeant d'un contenu tellement parodique qu'il met en lumière tout l'artifice des projets authentiques. En fait, ce qui est caché par la ribambelle des effets et des causes, c'est la prémisse et sa dimension aberrante, comme il en allait pour celle de Villaurrutia, personne ne s'étant inquiété de savoir d'où les miséreux tireraient les ressources nécessaires à payer chaussures et habits. Ici, au cas où

37. "Lo mismo digo de todas las demas cosas, y de todos los lugares; de suerte, que las harinas que se llevan a Veracruz, deberán ir primero a Zacatecas, y luego volverlas a traer para llevarlas a su destino. " Soit 1600 kilomètres. 
la parodie destructrice n'aurait pas été assez perceptible, l'auteur prend un plaisir manifeste à rajouter des propositions de nature à capter, par l'hilarité, des partisans à sa doucereuse mise en pièces. Faussement cordial, il ajoute donc :

¿Le parece a Vm. conveniente, que de cuando en cuando hubiese un atascadero, para obligar a los arrieros a que pidiesen auxilio al pueblo inmediato, y tuviesen este socorro los infelices de él? A mi me parece que sí: porque al fin el dinero se quedaba en casa, y esos mas se mantendrian.

Enfin, c'est le processus même de fièvre imaginative qui est supposé être à l'œuvre dans tout bon projet, transformé implicitement en activité pathologique, patelinement assimilé à un acte de déraison :

Una vez acalorada la imaginación, ocurren mil especies, que es difícil dexar de exponer: me parece que podría establecerse de cien en cien leguas, una compañia de salteadores, la qual obligase a los arrieros, a sacar tropa de la ciudad inmediata, que los defendiese, o a componerse con ellos amigablemente, dándoles una suma de consideración.

Ce petit chef d'œuvre de duplicité s'achevait sur la demande faite au rédacteur du Diario de dire "con ingenuidad su parecer", requête qui ne fut guère suivie d'effet. Ni réponse, ni commentaire ne suivirent et ce silence traduit parfaitement l'embarras insurmontable de ces ardents proyectistas qu'étaient les rédacteurs du Diario à trouver une réplique appropriée. De tous les discours polémiques, le plus efficace, finalement, se trouve être celui qui semble le plus étranger à l'esprit et à la lettre polémique, qui joue de l'insidieux et de la papelardise pour opérer la plus radicale des critiques.

Ici s'achève ce tour d'horizon d'un phénomène qui témoigne de la verve et de la vitalité des échanges dans cette société qui allait passer de la guerre des mots à la guerre tout court, une occupation où malheureusement le rire eut trop peu de place pour qu'il vaille la peine de s'en occuper. 